\title{
Dual role of transcription factor FoxO1 in controlling hepatic insulin sensitivity and lipid metabolism
}

\author{
Michihiro Matsumoto, Seongah Han, Tadahiro Kitamura, and Domenico Accili
}

Department of Medicine, Columbia University Medical Center, New York, New York, USA.

\begin{abstract}
Hepatic insulin resistance affects both carbohydrate and lipid metabolism. It has been proposed that insulin controls these 2 metabolic branches through distinct signaling pathways. FoxO transcription factors are considered effectors of the pathway regulating hepatic glucose production. Here we show that adenoviral delivery of constitutively nuclear forkhead box $\mathrm{O} 1$ (FoxO1) to mouse liver results in steatosis arising from increased triglyceride accumulation and decreased fatty acid oxidation. FoxO1 gain of function paradoxically increased insulin sensitivity by promoting Akt phosphorylation, while FoxO1 inhibition via siRNA decreased it. We show that FoxO1 regulation of Akt phosphorylation does not require DNA binding and is associated with repression of the pseudokinase tribble 3 (Trb3), a modulator of Akt activity. This unexpected dual role of FoxO1 in promoting insulin sensitivity and lipid synthesis in addition to glucose production has the potential to explain the peculiar admixture of insulin resistance and sensitivity that is commonly observed in the metabolic syndrome.
\end{abstract}

\section{Introduction}

Insulin resistance plays a fundamental role in the pathogenesis of a host of metabolic diseases, ranging from type 2 diabetes to hypertension, lipid disorders, atherosclerosis, and reproductive abnormalities (1). In liver, insulin resistance increases glucose production because of an impaired ability of insulin to suppress the expression/activity of gluconeogenic enzymes (2). This abnormality coexists with increased triglyceride (TG) synthesis (3) and reduced FFA oxidation (4), which are consistent with a heightened state of insulin sensitivity (5). This mixed picture of insulin sensitivity and resistance cannot be easily explained. It has been proposed that insulin controls glucose and lipid metabolism through different pathways, with insulin receptor substrate 2 (Irs2) acting on glucose production via forkhead box O1 (FoxO1) and Irs1 acting on lipid metabolism via sterol regulatory element-binding factor 1 (Srebf1) $(6,7)$ and FoxA2 (8). It should be noted that insulin's ability to regulate FoxA2 remains disputed (9).

Forkhead transcription factors of the FoxO subfamily (FoxOs) regulate metabolism, proliferation, and differentiation (10). In loss- and gain-of function experiments in mice, it has been shown that FoxO1 promotes hepatic glucose production $(11,12)$ and apoCIII expression (13). In liver, FoxO1 acts in concert with PPAR $\gamma$ coactivator $1 \alpha(\operatorname{Pgc} 1 \alpha)$ to stimulate glucose production through glucose-6-phosphatase, catalytic (G6Pc) and phosphoenolpyruvate carboxykinase 1 (Pck1), in cooperation with the cAMP/Creb pathway $(12,14)$. Insulin suppresses FoxO1 via phosphorylation-dependent nuclear exclusion. Fasting-induced gluconeogenesis prevents hypoglycemia, while the loss of insulin-dependent suppression of glucose production in diabetes causes fasting hyperglycemia (2).

Nonstandard abbreviations used: ChIP, chromatin immunoprecipitation; DBDFoxO1ADA, DNA binding-deficient FoxO1ADA mutant; DEX, dexamethasone; FoxO1, forkhead box O1; FSK, forskolin; Gsk3 $\alpha$, glycogen synthase kinase $3 \alpha$; G6Pc, glucose-6-phosphatase, catalytic; Irs2, insulin receptor substrate 2; Pck1, phosphoenolpyruvate carboxykinase 1; Pp2a-c, protein phosphatase 2a-c; Srebf1, sterol regulatory element-binding factor 1 ; TG, triglyceride; Trb3, tribble 3.

Conflict of interest: The authors have declared that no conflict of interest exists. Citation for this article: J. Clin. Invest. 116:2464-2472 (2006). doi:10.1172/JCI27047.
FoxO1 was originally identified as a negative regulator of insulin action in the roundworm Caenorbabditis elegans $(15,16)$, and its role in glucose production is fully consistent with that view. However, 2 newly emerged pieces of information led us to reassess FoxO1's hepatic role. The demonstration that FoxO1 promotes Irs 2 expression $(17,18)$ supports the possibility that FoxO1 regulates hepatic insulin sensitivity through a positive feedback mechanism. Moreover, conditional mutagenesis of Irs 1 and Irs 2 in liver indicated that neither substrate has a specific role in insulin action and that their actions are overlapping (19). Thus, we explored the possibility that FoxO1 is the shared element in insulin signaling that controls both lipid and glucose metabolism.

\section{Results}

Overexpression of constitutively nuclear FoxO1 in mouse liver. To investigate the effects of unmitigated FoxO1 activity, we introduced constitutively nuclear mutant FoxO1 (FoxO1ADA) (11) in mouse liver by injecting recombinant adenovirus. This mutant prevents insulin inhibition of Pck1 and G6pc expression (11, 12, 20). Four days after adenovirus delivery, FoxO1ADA was selectively expressed in liver and undetectable in other tissues (data not shown) (21). Unexpectedly, livers of mice transduced with FoxO1ADA were pale (Figure 1A); oil red $\mathrm{O}$ staining revealed the presence of diffuse intracellular lipid droplets (Figure 1B), and hepatic TG content increased 2-and 3-fold in the fasted and fed states, respectively (Figure 1C). Whereas transgenic mice overexpressing constitutively active FoxO1 develop impaired fasting glucose, hyperinsulinemia $(11,22)$, and increased TG levels (13), acute overexpression of FoxO1ADA reduced plasma insulin, glucose, and TG levels in the fed state, while total cholesterol levels increased slightly. Moreover, fasting $\beta$-hydroxybutyrate levels decreased by $90 \%$ in FoxO1ADA mice, compared with controls (Table 1). The decrease in fed insulin levels indicates that FoxO1ADA increased insulin sensitivity, thus accounting at least in part for lower glucose levels (see below). We measured expression of FoxO1 target genes, as well as genes involved in hepatic lipid metabolism. Consistent with previous gain-of-function experiments 
A
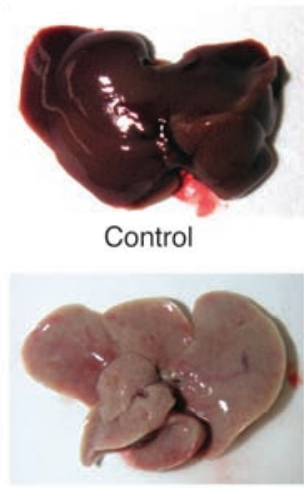

FoxO1ADA
B

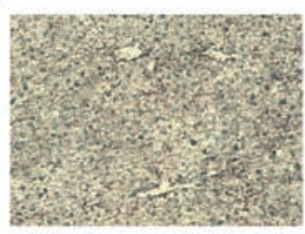

Control

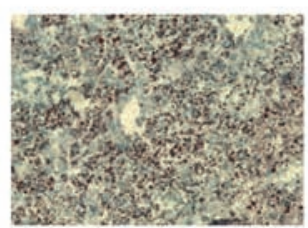

FoxO1ADA
C

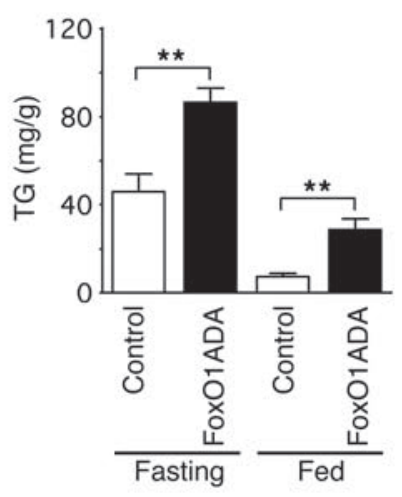

E
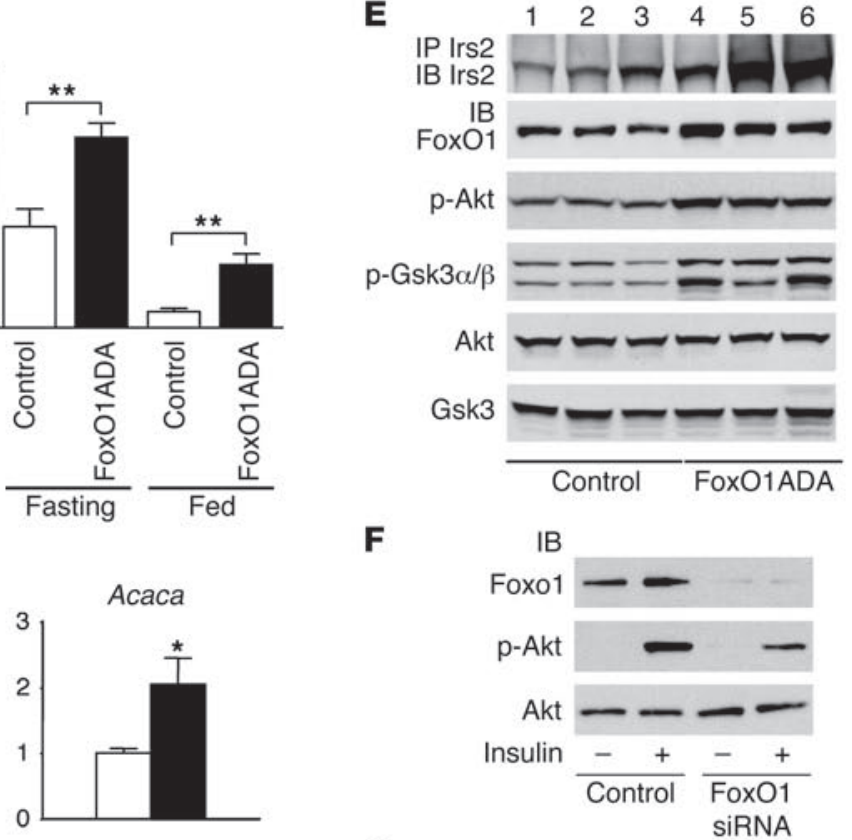

Acox 1

Fasn

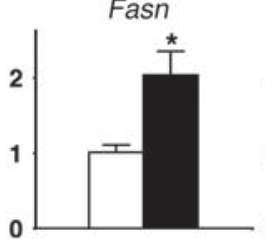

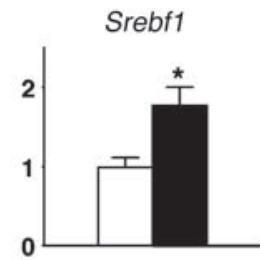

Ppara

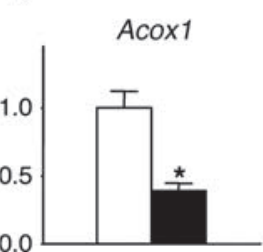

$\mathbf{F}$

IB

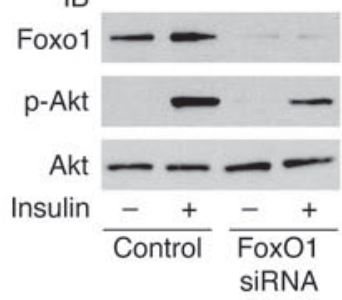

G

IB

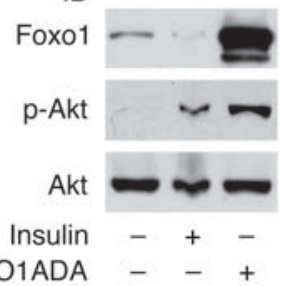

Figure 1

FoxO1 overexpression in liver. Macroscopic view of livers $(\mathbf{A})$ and oil red $\mathrm{O}$ staining of liver sections $(\mathbf{B})$ from representative mice injected with control empty adenovirus or adenovirus expressing FoxO1ADA and collected after an overnight fast. (C) Liver TG content in fasted and ad libitum-fed FoxO1ADA or control mice ( $n=6$ for each group). (D) Gene expression analysis by real-time RT-PCR in livers from ad libitum-fed control ( $n=6$; white bars) or FoxO1ADA mice $\left(n=6\right.$; black bars). Each PCR was carried out in triplicate. ${ }^{*} P<0.05$ and ${ }^{* *} P<0.01$. (E) Western blot analysis of liver extracts from adenovirus-injected mice. (F) Effect of FoxO1 inhibition by siRNA on insulin-induced Akt phosphorylation. We transduced cells with adenovirus encoding control siRNA or FoxO1siRNA and immunoblotted with the indicated antibodies. (G) Effect of FoxO1 DNA binding-deficient transduction on insulin-induced Akt phosphorylation. All data are representative of at least 3 independent experiments.

$(11,12,23)$, expression of IGF binding protein 1 (Igfbp1), G6pc, and Irs2 increased (Figure 1D). Moreover, G6pc and Pck1 mRNA levels were higher in the fed than in the fasted state, consistent with a loss of insulin-dependent inhibition (data not shown).

The increase in hepatic lipid content could be due to increased TG synthesis, decreased FFA oxidation, or both. We detected a 2-fold increase in expression of Srebf1 and its target genes fatty acid synthase (Fasn) and acetyl-coA carboxylase $\alpha$ (Acaca) and a 60\% decrease in expression of Ppara and its target gene acetyl-coA oxidase (Acox1) (Figure 1D). In contrast, expression of carnitine palmitoyl-transferase $1 \alpha(C p t 1 \alpha)$ was unchanged (data not shown). These data suggest that increased FoxO1 activity leads to lipid accumulation by activating TG synthesis through Srebf1 and inhibiting FFA oxidation through decreased expression of Ppara. The latter change provides a further explanation for the decrease in glucose levels, as mice lacking Ppara become hypoglycemic during fasting $(24,25)$.

FoxO1ADA increases Akt phosphorylation. The changes in Srebf1 and its transcriptional targets are surprising, as these genes are not known to be under FoxO1 control and are positively regulated by insulin in an Akt-dependent manner $(6,26)$. Thus, we measured activation of the Akt pathway. Indeed, we detected increased phosphorylation of both Akt and its substrates glycogen synthase kinase $3 \alpha$ (Gsk3 3 ) and Gsk $3 \beta$ in mice injected with FoxO1ADA, without changes in total Akt levels (Figure 1E, lanes 4-6). Next, we wanted to investigate whether FoxO1 loss of function would result in decreased Akt activity. Transduction of FoxO1-specific siRNA (FoxO1siRNA) adenovirus reduced endogenous FoxO 1 protein expression by approximately 95\% and impaired insulin-induced Akt activation by approximately $70 \%$ in primary hepatocytes (Figure $1 \mathrm{~F}$ ). Finally, we determined whether DNA binding by FoxO1 was required for its effect on Akt. We transduced hepatocytes with adenovirus encoding DNA binding-deficient FoxO1ADA mutant (DBD-FoxO1ADA) $(27,28)$.

DBD-FoxO1ADA induced basal Akt phosphorylation as effectively as FoxO1ADA (Figure 1G). These data indicate that FoxO1 regulates Akt phosphorylation by acting as a coregulator, rather than a transcription factor.

FoxO1ADA promotes Akt phosphorylation independent of insulin and Irs2. We investigated the mechanism of FoxO1-induced Akt activity in SV40-transformed mouse hepatocytes, an established model for studying hepatic insulin signaling (29). FoxO1ADA potenti- 
Table 1

Metabolic parameters

$\begin{array}{llcccc} & \begin{array}{l}\text { Insulin } \\ (\mathbf{n g} / \mathbf{m l})\end{array} & \begin{array}{c}\text { Glucose } \\ (\mathbf{m g} / \mathbf{d l})\end{array} & \begin{array}{c}\text { TG } \\ (\mathbf{m g} / \mathbf{d l})\end{array} & \begin{array}{c}\text { Total cholesterol } \\ (\mathbf{m g} / \mathbf{d l})\end{array} & \begin{array}{c}\beta-0 H \text { butyrate } \\ (\mathbf{m M})\end{array} \\ \text { Control (empty) }(n=6) & 2.6 \pm 0.6 & 110 \pm 8 & 27 \pm 1 & 59 \pm 3 & 0.27 \pm 0.03 \\ \text { Fox01ADA }(n=6) & 0.7 \pm 0.1^{\mathrm{A}} & 65 \pm 8^{\mathrm{A}} & 12 \pm 1^{\mathrm{A}} & 77 \pm 6^{\mathrm{A}} & 0.06 \pm 0.01^{\mathrm{B}}\end{array}$

Metabolic parameters in mice injected with control (empty) or FoxO1ADA adenovirus. Samples were collected in fed (for measurement of glucose and insulin) or 6-hour fasted mice (for measurement of TG, cholesterol, and $\beta$ - $\mathrm{OH}$ butyrate). ${ }^{A} P<0.01$. ${ }^{\mathrm{B}} P<0.05$. induced Irs2 expression in a dose-dependent manner (Figure 2A). Higher levels of FoxO1ADA overexpression induced basal Akt and Gsk3 phosphorylation to levels comparable to those seen in response to insulin (Figure $2 \mathrm{~B}$ ).

We tested whether induction of Akt phosphorylation by FoxO1ADA is due to increased Irs 2 expression. The next 2 experiments provided evidence disproving this possibility. In the first experiment, we took advantage of the observation that prolonged hyperinsulinemia decreases Irs2 expression $(6,30)$. We compared the ability of FoxO1ADA and Irs2 to increase Akt phosphorylation in a cellular model of chronic hyperinsulinemia. In hepatocytes, prolonged insulin treatment decreased expression and tyrosine phosphorylation of Irs 2 and reduced Akt phosphorylation by insulin (Figure 2C, compare lanes 1 and 3 and lanes 2 and 4). Expression of FoxO1ADA restored Akt phosphorylation to approximately $50 \%$ of the levels seen in insulin-naive cells, while Irs2 overexpression was unable to do so (Figure 2D, compare lanes 2,4 , and 6 , panel marked by arrow). Next, we measured the effects of FoxO1ADA in $\mathrm{C}_{2} \mathrm{C}_{12}$ myotubes. In these cells, Irs1 is expressed at higher levels than Irs2 (Figure 2E, bottom 2 panels). Transduction of FoxO1ADA dose-dependently induced Akt and Gsk3 phosphorylation to levels similar to those induced by insulin but failed to increase Irs2 levels. Irs1 expression was also unaffected (Figure 2E, lanes 3-5). The combined data in hepatocytes and myotubes suggest that FoxO1-induced Akt phosphorylation is independent of Irs2. ated insulin-induced phosphorylation of Akt and Gsk3 $\alpha / \beta$ and
A ( fected by FoxO1ADA, nor were the amounts of Pdk1 and protein phosphatase 2a-c (Pp2a-c), both of which can directly regulate Akt phosphorylation (31) (Figure 3B). Thus, FoxO1-induced Akt activation is due neither to increased receptor tyrosine kinase activity, phosphatidylinositol 3,4,5-trisphosphate $\left(\mathrm{PIP}_{3}\right)$ levels, and Pdk1 activity nor to decreased $\mathrm{Pp} 2 \mathrm{a}-\mathrm{c}$ activity.

FoxO1ADA does not affect Akt kinases and lipid or protein phosphatases. Akt is regulated by multiple factors (31). To investigate the mechanism(s) of FoxO1-mediated Akt activation, we examined pathways involved in Akt activation. FoxO1ADA induced Akt phosphorylation in hepatocytes lacking insulin receptors, indicating that the insulin receptor is not necessary for this event (Figure 3A). Similarly, FoxO1ADA promoted Akt phosphorylation without increasing binding of the p85 subunit of PI3K horylated proteins (Figure 3B). Expression of

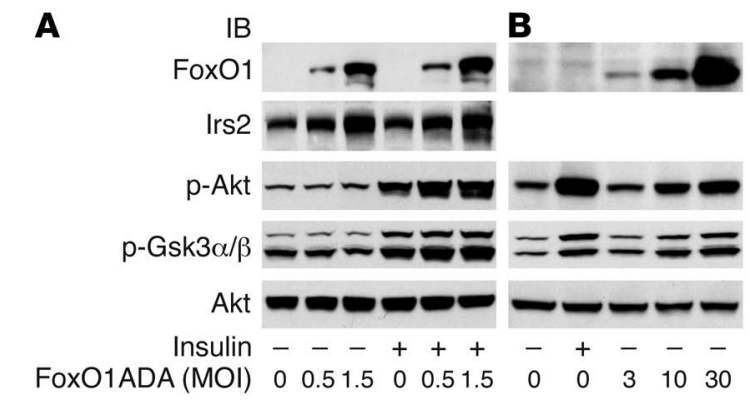

C

CoxO1ADA adenovirus D Irs2 adenovirus $\begin{array}{llllllllllll}1 & 2 & 3 & 4 & 5 & 6 & 1 & 2 & 3 & 4 & 5 & 6\end{array}$

IB: FoxO1 - - -

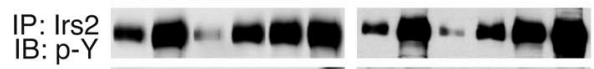

IP: Irs2 Orse Irs2

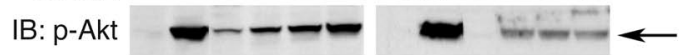

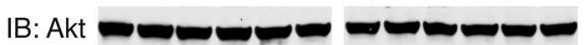

$\mathrm{IB}: \mathrm{p}-\mathrm{Gsk} \beta \alpha / \beta \quad$ ニニニニ ニ-ニニニ

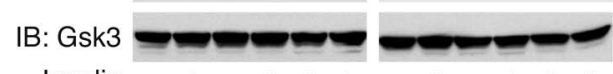

Insulin -+-+++-+-+++

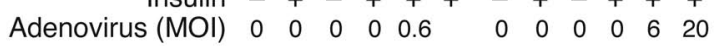
Insulin pretreatment --++++-++++

(100 nM, 24 h)

\section{Figure 2}

FoxO1ADA promotes Akt activation independent of increased Irs2 expression. (A and B) Effects of FoxO1ADA in SV40-transformed hepatocytes transduced with FoxO1ADA at different MOIs. We incubated cells with insulin for 10 minutes and subjected cell lysates to immunoblot analysis with the indicated antibodies. All data are representative of at least 3 independent experiments. Fao hepatoma cells transduced with FoxO1ADA (C) or Irs2 (D) adenovirus were preincubated for 24 hours in the absence or presence of insulin. For immunoprecipitation, cells were treated for 2 minutes with insulin, and lysates were immunoprecipitated with antibodies against Irs2 and immunoblotted with either anti-phosphotyrosine or anti-Irs2 antibodies. For direct immunoblot analysis, the immunoprecipitation step was omitted. All data are representative of at least 2 independent experiments. (E) $\mathrm{C}_{2} \mathrm{C}_{12}$ myotubes transduced with FoxO1ADA adenovirus were incubated with insulin for 10 minutes and analyzed by direct immunoblotting with the indicated antibodies or immunoprecipitated and immunoblotted with antibodies to either Irs1 or Irs2. 
A

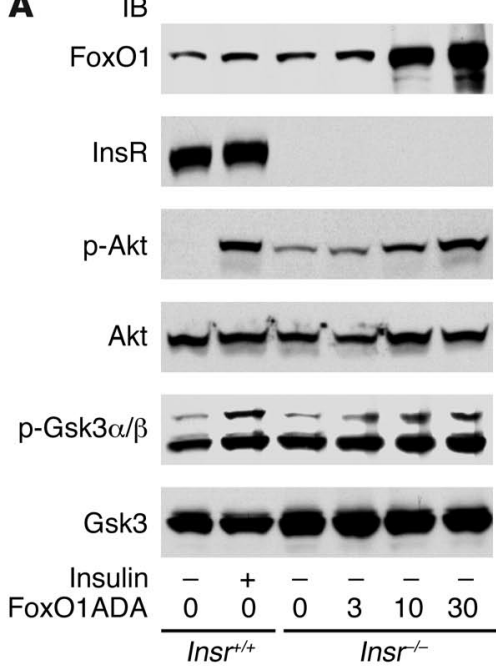

B

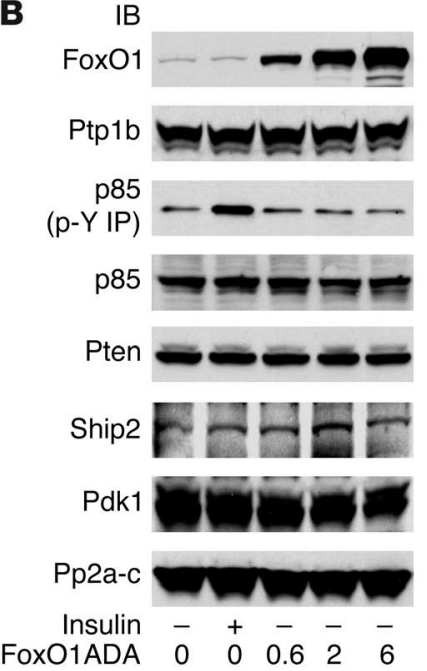

C

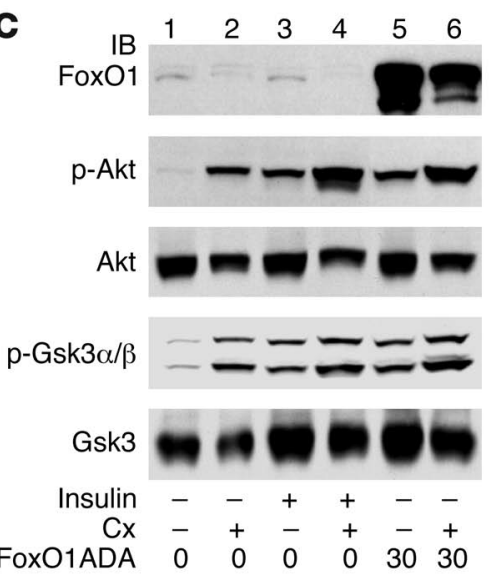

Figure 3

FoxO1ADA does not affect expression of Akt pathway components in hepatocytes. (A) SV40 hepatocytes from WT (Insr+/+) or insulin receptor-deficient $\left(I n s r^{--}\right)$mice transduced with FoxO1ADA adenovirus were incubated with insulin, lysed, and subjected to immunoblot analysis with the indicated antibodies. (B) Fao cells transduced with FoxO1ADA adenovirus were incubated in the absence or presence of insulin for 2 minutes and then lysed. Cell lysates were either immunoblotted with the indicated antibodies or immunoprecipitated with antibody against anti-phosphotyrosine ( $p$ - $Y$ IP) and immunoblotted with antibody against the PI3K p85 subunit. (C) Hepatocytes transduced with FoxO1ADA adenovirus were preincubated with cycloheximide $(\mathrm{Cx})$ for 16 hours. Thereafter, cells were treated with insulin, lysed, and analyzed by immunoblot with the indicated antibodies.

To determine whether FoxO1ADA-dependent Akt phosphorylation required new protein synthesis, we examined the effect of cycloheximide on this process. Surprisingly, cycloheximide treatment induced Akt phosphorylation in serum-deprived cells (Figure 3C, lanes 1 and 2), to levels comparable to those induced by insulin treatment (Figure 3C, lanes 3 and 4). These data suggest that negative modulators of Akt are suppressed by inhibition of protein synthesis. We could not evaluate the combined effects of FoxO1ADA and cycloheximide, because the latter decreased FoxO1ADA expression (Figure 3C, lanes 5 and 6). Nonetheless, these data prompted us to ask whether FoxO1 acted by preventing expression of Akt inhibitors.

FoxO1 suppresses Trb3 expression in an insulin-sensitive manner. The pseudokinase tribble 3 (Trb3) binds Akt and prevents its phosphorylation by insulin (32). Fasting and diabetes promote Trb3 expression through the $\mathrm{Creb} / \operatorname{Pgc} 1 \alpha / \mathrm{P}$ par $\alpha$ pathway (32), while inhibition of Trb3 by siRNA improves glucose tolerance (33). Thus, Trb3 is a plausible candidate to modify Akt activity in response to FoxO1. We investigated the effect of FoxO1ADA on Trb3 mRNA expression in Fao hepatoma cells. Forskolin plus dexamethasone (FSK-DEX) and insulin increased Trb3 expression, and FoxO1ADA suppressed it in a dosedependent manner independent of FSK-DEX or insulin (Figure 4A, upper panel). The increase in Trb3 expression in response to insulin is somewhat surprising, given that Trb3 is suppressed by feeding in vivo (32). These data appear to indicate that factors other than insulin are responsible for postprandial Trb3 inhibition. As a positive control for FoxO1 function, we showed that FoxO1ADA induced G6pc expression $(11,12)$ and prevented insulin inhibition of G6pc expression induced by FSK-DEX $(12,20)$ (Figure 4A, middle panel).

To confirm the involvement of the PI3K/Akt pathway in insulin regulation of Trb3 expression, we examined the effects of PI3K inhibitors and of constitutively active mutants of PI3K (Myr-p110) and Akt (Myr-Akt) on Trb3 mRNA. Treatment with the PI3K inhibitor LY294002 decreased Trb3 and increased G6pc expression (Figure 4B, lane 3). Conversely, Myr-p110 (Figure 4B, lanes 5 and 6) and MyrAkt (Figure 4C, lanes 3 and 4) promoted Trb3 and inhibited G6pc expression. FoxO1ADA blocked the effect of insulin (Figure 4B, lane 4), Myr-p110 (Figure 4B, lane 6), and Myr-Akt (Figure 4C, lane 4) on Trb3 mRNA. These data indicate that FoxO1 decreases Trb3 expression, whereas activation of PI3K promotes it.

The decrease in Trb3 mRNA was paralleled by a decrease in Trb3 protein expression (Figure 4D, upper panel). In contrast, Irs2 overexpression failed to bring about changes in Trb3 protein expression (Figure 4D, lower panel). Further, Trb3 expression was inhibited by cycloheximide (Figure 4E). These results support the conclusions derived from experiments represented in Figure 2, C and D, and Figure 3C. We also examined Trb3 expression in livers of mice transduced with FoxO1ADA. Consistent with data in cultured cells, Trb3 mRNA expression decreased by approximately $80 \%$ (Figure 4F) and $\mathrm{Trb} 3$ protein expression by more than $90 \%$ (Figure $4 \mathrm{G}$ ) in these mice. We conclude that FoxO1 suppresses Trb3 expression.

FoxO inbibits Trb3 independent of Pgc1 $\alpha$ and Ppar $\alpha$. Pgc1 $\alpha$ is a key FoxO1 coactivator in hepatic gluconeogenesis (12) and increases Trb3 expression by coactivating PPAR $\alpha$ (33). Thus, the decrease in Trb3 could be due to decreased Pgc1 $\alpha$ expression, but FoxO1ADA overexpression (2- to 3-fold above endogenous levels; Figure 1E) failed to affect Pgc1 $\alpha$ levels (data not shown). We next tested whether FoxO1ADA inhibited Trb3 by competing with Ppar $\alpha$ for a limited pool of their shared coactivator Pgc1 $\alpha$. To examine this possibility, we asked whether Pgc1 $\alpha$ overexpression rescued FoxO1ADA's inhibition of Trb3 expression. FoxO1ADA induced a 50\% $\pm 10 \%$ decrease in Trb3 expression in Fao cells (Figure 4H, lane 4 versus 1). Pgc1 $\alpha$ increased Trb3 levels by $20 \% \pm 5 \%$ (Figure $4 \mathrm{H}$, lanes 2 and 3 ), and cotransduction of FoxO1ADA and Pgc1 $\alpha$ resulted in a 50\% $\pm 10 \%$ decrease in Trb3 expression, similar to what occurred in cells expressing FoxO1 alone. Thus, the effect of FoxO1ADA on Trb3 is unlikely to be 
A

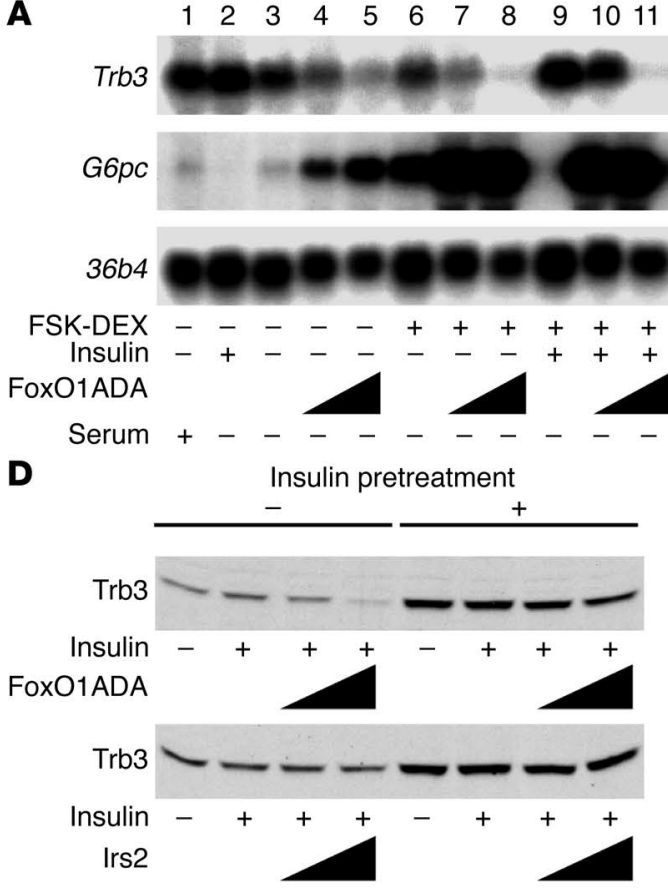

B

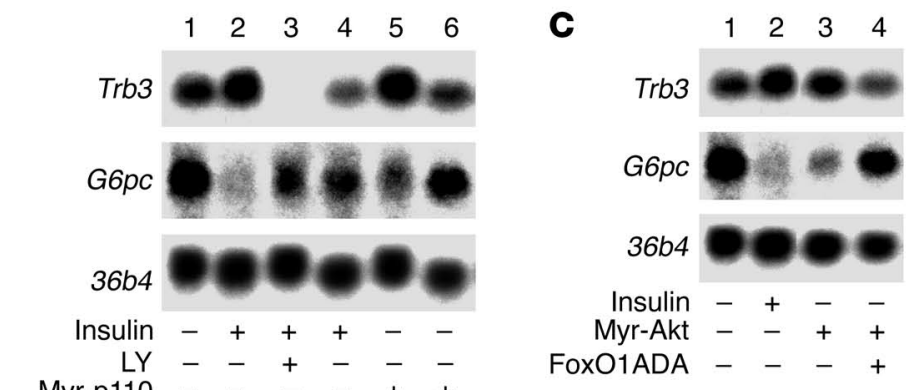

E
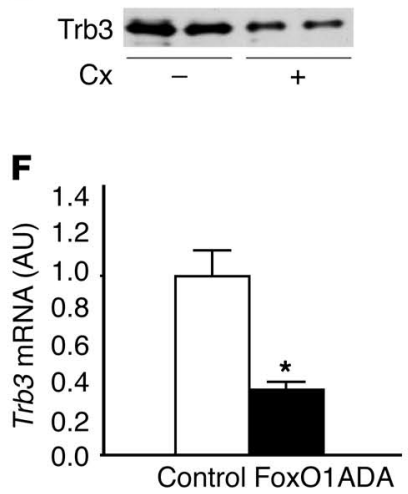

G

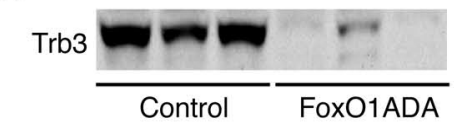

H

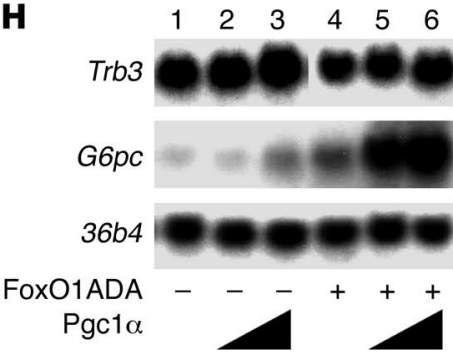

\section{Figure 4}

FoxO1 suppresses Trb3 expression in an insulin-dependent manner. Northern blot analyses of Fao cells transduced with FoxO1ADA adenovirus and incubated with FSK-DEX and/or insulin for 20 hours (A); FoxO1ADA and Myr-p110 and treated with insulin in the absence or presence of LY294002 (LY) (B); FoxO1ADA and Myr-Akt and treated with or without insulin (C). 36b4 is a housekeeping gene used as a control for gel loading. (D) Hepatocytes transduced with FoxO1ADA (upper panel) or Irs2 (lower panel) adenoviruses were preincubated with insulin. Thereafter, cells were treated for 10 minutes with fresh insulin-containing medium, lysed, and immunoblotted with the indicated antibodies. All data are representative of at least 2 independent experiments. (E) Immunoblot analysis of the effect of cycloheximide on Trb3 expression. (F) Gene expression analysis by real-time RT-PCR in livers of control or FoxO1ADA mice ( $n=6$ for each). Each PCR was carried out in triplicate. ${ }^{*} P<0.05$. (G) Western blot analysis of Trb3 expression in liver from control or FoxO1ADA mice. Northern blot and immunoblot data are representative of 3 independent experiments. (H) Northern blot analyses of Fao cells transduced with FoxO1ADA and Pgc1 $\alpha$.

due to sequestration of Pgc1 $\alpha$ from Ppar $\alpha$. In contrast, Pgc1 $1 \alpha$ expression had a synergistic effect with FoxO1ADA to increase G6pc mRNA expression (Figure 4H, lanes 4-6), consistent with previous observations (12). These findings indicate that FoxO1 inhibits Trb3 expression independent of Pgc1 $\alpha$.

Ppara regulates Trb3 expression and is inhibited by FoxO1ADA. Thus, we asked whether FoxO1 regulation of Trb3 is mediated by the same cis-acting DNA elements required for Pparo-dependent transcription. A $2.7-\mathrm{kb}$ promoter fragment possessed strong promoter activity (Figure 5A, lane 2), which was further increased by FSX-DEX (Figure 5A, lane 4), insulin (Figure 5A, lane 5), or a combination of both (Figure 5A, lane 6) and was inhibited by LY294002 (Figure 5A, lanes 3 and 7). Transfection or expression of FoxO1ADA suppressed both basal (Figure 5B, lanes 4 and 5) and insulin-stimulated Trb3 activity (Figure 5B, lane 6). WT FoxO1 had a similar but less pronounced effect (Figure 5B, lanes 7-9). In addition, FoxO1ADA inhibited Trb3 promoter activity induced by Myr-p110 and Myr-Akt (Figure 5C, black bars), while WT FoxO1 had a smaller effect on basal and Myr-p110-dependent activity and no effect on Myr-Akt-dependent activity (Figure 5C, gray bars). These data indicate that FoxO1 inhibits Trb3 promoter activity in an insulin-sensitive manner via the PI3K/Akt pathway.
To identify the sequence responsible for transcriptional inhibition of Trb3 promoter by FoxO1, we performed promoter deletion studies and chromatin immunoprecipitation (ChIP) assays. A minimal Trb3 promoter $(-339$ to +104$)$ retained full activity and was inhibited by LY294002 and FoxO1ADA (Figure 5D and Supplemental Figure 1A; supplemental material available online with this article; doi:10.1172/JCI27047DS1). Because this promoter does not contain the Ppar $\alpha$ response element, we conclude that FoxO1 can inhibit Trb3 independent of its effect on Ppara. Indeed, ChIP revealed that FoxO1 binds to this sequence and that insulin inhibits binding of WT FoxO1 but not FoxO1ADA (Figure 5E). This region contains 2 putative forkhead consensus binding sites (Supplemental Figure 1B). However, individual (data not shown) and combined mutations of these sites did not affect the ability of LY294002 and FoxO1ADA to inhibit promoter activity (Figure 5F and Supplemental Figure 1B). This is consistent with the observation (Figure $1 \mathrm{G}$ ) that FoxO1 controls Akt activity in a DNA binding-independent manner. To investigate the requirement of FoxO1 in Trb3 transcription, we examined the effect of FoxO1siRNA on Trb3 promoter activity in SV40 hepatocytes. While FoxO1siRNA increased promoter activity (Figure 5G), it failed to affect Trb3 levels in primary hepatocytes (data not shown). These data suggest that loss of FoxO1 function is 

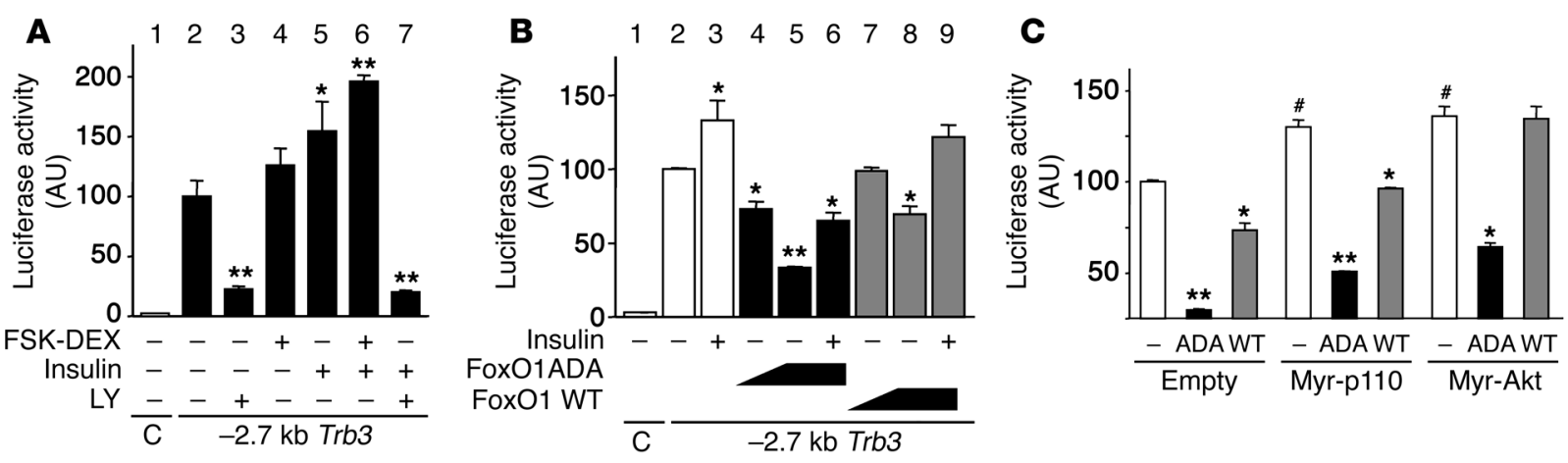

D

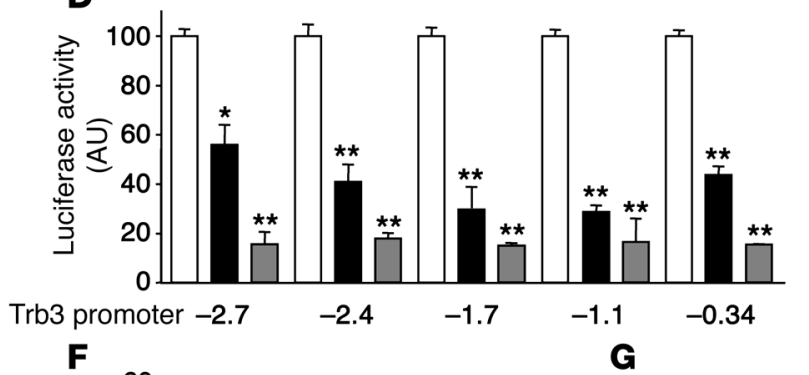

E
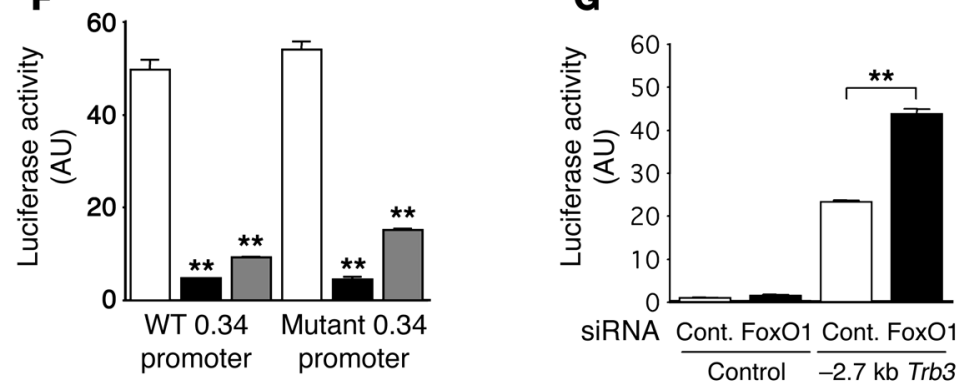

Figure 5

FoxO1 regulation of Trb3 promoter activity. (A) SV40 hepatocytes were transiently cotransfected with reporter plasmids and incubated with FSK-DEX and/or insulin or LY294002. (B) Eight hours after cotransfection, cells were incubated with insulin for 16 hours. (C) Cells were transduced with either empty, Myr-p110, or Myr-Akt adenovirus. After 24 hours, cells were transiently cotransfected with the indicated plasmids and adenoviruses. (D) Deletion analysis of the Trb3 promoter in SV40 hepatocytes cultured in the presence of FSK-DEX (white bars), FoxO1ADA (black bars), or LY294002 (gray bars). ${ }^{*} P<0.05$ vs. vector-transfected cells; ${ }^{* \star} P<0.01$; and ${ }^{\#} P<0.05$ vs. $\beta$-gal-transduced cells. (E) ChIP in SV40 hepatocytes. We immunoprecipitated the following with anti-FoxO1 (upper panel) or control antiserum (middle panel): lane 1, untransduced cells; lane 2, cells transduced with FoxO1ADA; lanes 3-4, cells transduced with WT FoxO1. Bottom panel: total input DNA. (F) Mutation analysis. Control (WT) or mutant Trb3 promoter with mutations of the Foxo sites (Mutant) were assayed in basal conditions (white bars) and in the presence of LY294002 (black bars) or FoxO1ADA (gray bars). ${ }^{* *} P<0.01$. (G) Effect of FoxO1 knockdown on Trb3 promoter activity. SV40 hepatocytes were transiently cotransfected with either FoxO1siRNA or control siRNA with Trb3 promoter reporter construct at final concentration of $30 \mathrm{nM}$. ${ }^{\star \star} P<0.01$.

necessary but not sufficient to promote Trb3 expression. Additional signal(s) downstream of PI3K or changes in nutrient availability (34) may also be required for Trb3 regulation.

\section{Discussion}

FoxO1 participates in feedback regulation of insulin signaling. Using gainand loss-of-function approaches in mice and isolated hepatocytes, we show that, in addition to its previously demonstrated role in hepatic glucose production $(11,12)$, FoxO1 provides positive feedback to fine-tune insulin sensitivity via increased Akt phosphorylation. What is the physiologic significance of this mechanism? Positive feedback by FoxO1 would allow a rapid response to changes in nutrients' availability and hormonal environment during the transition from fasting to feeding and enable effective nutrient storage in response to insulin. In this regard, there is an intriguing analogy with the
Drosophila FoxO1 ortholog (dfoxo). Dfoxo activates transcription of both proximal and distal components of the insulin receptor (dir) signaling cascade to control growth in response to nutrient availability, including the translational repressor $d 4 e b p$ and $\operatorname{dir}(35)$. When nutrients are limited, $d f o x o$ increases dir expression to prime for changes in nutrient availability and $d 4$ ebp expression to inhibit growth. Dfoxo's control of multiple steps in the insulin signaling pathway may represent an evolutionarily conserved function with mammalian FoxO1.

There are likely to be multiple mechanisms by which FoxO1 increases insulin sensitivity during fasting. Reduced Ppar $\alpha$ expression has a profound metabolic impact and is thus probably related to the phenotype observed in FoxO1ADA mice $(24,25$, 36). Trb3 expression has been proposed to regulate insulin sensitivity via Akt inhibition. Trb3 is induced during fasting by Pgc1 $\alpha$ coactivation of Ppar $\alpha(32,33)$. Since Pgc1 $\alpha$ also coactivates 
FoxO1 (12), it could have been predicted that FoxO1 increases Trb3 expression. Surprisingly, our data show that: (a) insulin induces and (b) FoxO1 inhibits Trb3 expression; moreover, (c) the cis-acting elements in the Trb3 promoter required for FoxO1 repression are distinct from those utilized by Pgc1 $\alpha / \operatorname{Ppar} \alpha$ (33). The simplest explanation of our data is that FoxO1ADA suppresses Trb3 by at least 2 independent mechanisms: decreased Ppar $\alpha$ expression and trans-repression. The trans-repressor function of FoxO1 is independent of direct binding to the Trb3 promoter and is thus likely to result from protein-protein interactions with other components of the transcriptional apparatus. The presence of multiple mechanisms by which FoxO1 modulates insulin sensitivity can also explain why FoxO1 ablation did not result in increased Trb3 levels, even as it lowered Akt phosphorylation. The link between hepatic FoxO1 and Trb3 regulation in disease states is an important area of future endeavors.

The objection could be raised that FoxO1-induced Akt activity would in turn cause FoxO1 phosphorylation and nuclear exclusion, resulting in a futile cycle. But 3 points should be considered. First, FoxO1-induced Akt phosphorylation is independent of DNA binding. Thus, even a phosphorylated FoxO1 would retain the ability to induce Akt by acting as a trans-repressor. This would be consistent with the recent demonstration, by us as well as others, that other posttranslational modifications, such as acetylation, can override phosphorylation as a targeting signal $(37,38)$. Second, we have previously shown that Akt phosphorylation is necessary but not sufficient for full inactivation of FoxO1 $(39,40)$. Third, one would indeed predict that this mechanism should be to some extent self-limiting; otherwise, unchecked increases in insulin sensitivity would cause hypoglycemia during fasting.

FoxO overactivity and the metabolic syndrome. The liver plays a key role in the development of the metabolic sequelae of insulin resistance. Insulin resistance increases glucose production because of an impaired ability of insulin to suppress expression and/or activity of gluconeogenic enzymes (2). At the same time, there is increased TG synthesis, which is consistent with a heightened state of insulin sensitivity; and decreased FFA oxidation, which is intertwined with glucose oxidation (5). This mixed picture of insulin sensitivity and resistance cannot be easily explained. It has been proposed that in other insulin target cells, such as vascular endothelial (41) or ovarian granulosa cells (42), elevated plasma insulin concentrations lead to activation of the IGF-1 receptor or of hybrid insulin/IGF-1 receptors (43). However, this explanation falls short in the liver, which lacks IGF-1 receptors. It has been suggested that hepatic lipid abnormalities result from selective intracellular insulin sensitivity such that, while insulin signaling to glycogen storage and glucose release is impaired, signaling to lipid synthesis is sensitive. But evidence to support intracellular specificity of insulin signaling remains indirect (7, 21, 29, 44, 45).

The present study provides a parsimonious mechanism to explain the pathogenesis of the metabolic syndrome in liver. We propose that, regardless of which Irs, PI3K, and Akt isoforms are activated in response to insulin, all these branches converge upon FoxO proteins. Thus, in conditions of impaired insulin signaling, FoxO activity increases, leading to excessive glucose production (23). At the same time, it increases Akt signaling and suppresses Ppara expression, leading to increased TG synthesis and decreased fatty acid oxidation. This mechanism could sustain a mixed state of resistance and sensitivity to insulin. We speculate that the dual role of FoxO1 in liver can be explained by its ability to function as a transcription factor or as a coregulator, with different effects on different target genes. Further studies will be required to test the relevance of our model to the metabolic syndrome.

\section{Methods}

Chemicals and antibodies. We obtained insulin, FSK, DEX, and cycloheximide from Sigma-Aldrich; LY294002 from Calbiochem, EMD Biosciences; polyclonal antibodies against phospho-Ser473Akt, Akt, phospho-Gsk $\alpha / \beta$ (Ser21/9), and Pdk1 from Cell Signaling Technology; polyclonal antibodies against FoxO1 and insulin receptor $\beta$ subunit from Santa Cruz Biotechnology Inc.; polyclonal antibodies against Irs1, Irs2, p85, Ptp1b, Ship2, phosphotyrosine (4G10), and Pp2a-c subunit from Upstate USA Inc.; monoclonal antibodies against Gsk $3 \beta$ from BD Biosciences; and polyclonal antibodies against Pten from Cascade BioScience. The anti-Trb3 antiserum has been described previously (32).

Cell culture. We cultured Fao hepatocytes in RPMI 1640 medium supplemented with $100 \mathrm{U} / \mathrm{ml}$ penicillin, $100 \mu \mathrm{g} / \mathrm{ml}$ streptomycin, and $10 \%$ fetal calf serum under an atmosphere of $5.0 \% \mathrm{CO}_{2} . \mathrm{C}_{2} \mathrm{C}_{12}$ myoblasts were grown at $37^{\circ} \mathrm{C}$ in high-serum growth medium containing DMEM with $4.5 \mathrm{~g} / \mathrm{l}$ glucose, $100 \mathrm{U} / \mathrm{ml}$ penicillin, $100 \mu \mathrm{g} / \mathrm{ml}$ streptomycin, and $10 \%$ fetal calf serum under an atmosphere of $7.5 \% \mathrm{CO}_{2}$. Myoblasts were switched from high-serum growth medium to low-serum differentiation medium (DMEM with $4.5 \mathrm{~g} / 1$ glucose, $100 \mathrm{U} / \mathrm{ml}$ penicillin, $100 \mu \mathrm{g} / \mathrm{ml}$ streptomycin, and $2 \%$ horse serum) to induce myogenic differentiation (46). SV40-transformed hepatocytes were cultured as describe previously (20). Primary mouse hepatocytes were isolated from 8- to 12-week-old male C57BL/6 mice as described previously (26) and cultured in Medium 199 (Invitrogen) with 5\% FBS at 5\% CO 2 . Cells were incubated in serum-free Medium 199 overnight before addition of insulin.

Adenoviral vectors and adenoviral transduction. Adenoviral vectors encoding constitutively active PI3K (Myr-p110), Akt (Myr-Akt) (47), and FoxO1 (ADA) (20), as well as WT Pgc1 $\alpha$ (12) and LacZ were described previously (13). For knockdown of FoxO1 in hepatocytes, we used DNA-based adenoviral vector-mediated technology (Knockout Adenoviral RNAi System 2; BD Biosciences), with GCACCGACTTTATGAGCAACC as the targeted sequence. We used negative control short-hairpin RNA sequence (BD Biosciences) as a control siRNA target sequence. We generated recombinant adenovirus expressing mouse Irs2, DBD-FoxO1ADA, control siRNA, or FoxO1siRNA using Adeno-X Expression System 2, purified it with Adeno-X Virus Purification Mega Kit, and titrated it with Adeno-X Rapid Titer Kit (all from BD Biosciences). We transduced Fao or SV40-transformed hepatocytes at the indicated MOIs by incubation for 3 hours at $37^{\circ} \mathrm{C}$ in either RPMI 1640 or $\alpha$-MEM medium supplemented with $0.5 \%$ BSA. The virus-containing medium was then aspirated, and cells were incubated for an additional 30-34 hours in RPMI 1640 supplemented with $10 \%$ fetal calf serum. For Northern blot analysis, cells were preincubated in 0.5\% BSA-containing RPMI 1640 for 16 hours, then treated for 20 hours with FSK $(10 \mu \mathrm{M})$, DEX (100 nM), insulin $(100 \mathrm{nM}), \mathrm{LY} 294002(30 \mu \mathrm{M})$, or cycloheximide $(5 \mu \mathrm{M})$.

RNA isolation and expression studies. We extracted RNA using RNeasy Mini Kit and RNase-Free DNase Set (QIAGEN). We carried out Northern hybridization as described previously (7). We used NIH Image 1.63 software (http://rsb.info.nih.gov/nih-image/download.html) for quantification of Northern blot analysis. We obtained a Trb3 probe by amplification of mouse genomic DNA. PCR primers were: 5 '-CTCTGAGGCTCCAGGACAAG-3' and 5'-GGACTGCTAGGGAAGGAAGG-3'. For real-time PCR analysis, we reverse transcribed total RNA using SuperScript II First-Strand Synthesis System (Invitrogen). The sequences of oligonucleotide primers employed were: $18 s, 5^{\prime}$-AAACGGCTACCACATCCAAG-3' and 5'-CCTCCAATGGATCCTCGTTA-3'; Irs2, 5' -TCCAGAACGGCCTCAACTAT- $3^{\prime}$ and 5'-AGTGATGGGACAGGAAGTCG-3'; G6pc, 5'-GCTTGGATTCTACCTGCTAC-3' and $5^{\prime}$-AAAGACTTCTTGTGTGTCTGTC-3'; Igfbp1, 5' -AGATC- 
GCCGACCTCAAGAAAT-3' and 5'-CTCCAGAGACCCAGGATTTT-3'; Srebf1, 5'-GAAGCTGTCGGGGTAGCGTCT-3' and 5'-CTCTCAGGAGAGTTGGCACCTG-3'; Fasn, 5'-GCTGCGGAAACTTCAGGAAAT-3' and 5'-AGAGACGTGTCACTCCTGGACTT-3'; Acaca, 5'-GGACAGACTGATCGCAGAGAAAG-3' and 5'-GCTGTTCCTCAGGCTCACAT-3'; Ppara, 5'-GGGTACCACTACGGAGTTCACG-3' and 5'-CAGACAGGCACTTGTGAAAACG-3'; Acox1, 5'-GTGCAGCTCAGAGTCTGTCCAA$3^{\prime}$ and $5^{\prime}$-TACTGCTGCGTCTGAAAATCCA-3'; Cpt1a, 5' -TGCACTACGGAGTCCTGCAA-3' and 5'-GGACAACCTCCATGGCTCAG-3'; and Trb3, $5^{\prime}$-CTGTGAGAGGACGAAGCTGGTG- ${ }^{\prime}$ and $5^{\prime}$-AAGGTCCCTCTACGGATCTTGC-3'. PCR reactions were performed in triplicate using a DNA Engine Opticon 2 System (MJ Research). Relative mRNA levels were calculated using a standard curve, with the PCR product for each primer set normalized to $18 \mathrm{~S}$ ribosomal RNA.

Immunoprecipitation and Western blotting. We lysed cells in RIPA buffer (50 mM Tris- $\mathrm{HCl}, \mathrm{pH} 7.5,150 \mathrm{mM} \mathrm{NaCl}, 0.1 \% \mathrm{SDS}, 1 \% \mathrm{Na}$ deoxycholate, $1 \%$ NP-40, $10 \mathrm{mM} \mathrm{NaF}, 5 \mathrm{mM} \mathrm{Na} V_{4}, 2 \mathrm{mg} / \mathrm{ml}$ pepstatin, $2 \mathrm{mM}$ PMSF, $1 \mathrm{mM}$ DTT, $20 \mu \mathrm{g} / \mathrm{ml}$ leupeptin, and $10 \mu \mathrm{g} / \mathrm{ml}$ aprotinin). For immunoprecipitation, we lysed cells or liver extracts in buffer containing $20 \mathrm{mM}$ Tris- $\mathrm{HCl}$ (pH 7.5), 1\% NP-40, 10\% glycerol, $137 \mathrm{mM} \mathrm{NaCl}$, $1 \mathrm{mM} \mathrm{MgCl}_{2}, 2.5 \mathrm{mM} \mathrm{CaCl}_{2}, 1 \mathrm{mM} \mathrm{DTT}, 1 \mathrm{mM}$ PMSF, $1 \mathrm{mM} \mathrm{Na} \mathrm{VO}_{4}$, $1 \mathrm{mM}$ EDTA, $50 \mathrm{mM} \mathrm{NaF}, 10 \mathrm{mM} \mathrm{NaPPi}, 2 \mu \mathrm{g} / \mathrm{ml}$ leupeptin, and $10 \mu \mathrm{g} / \mathrm{ml}$ aprotinin. The lysates were subjected to immunoprecipitation, resolved on SDS-PAGE, transferred onto nitrocellulose membranes, and probed with the various antibodies. Immunoreactive proteins were visualized with ECL Western blotting detection reagents (Amersham Bioscience).

Plasmid construction. We amplified Trb3 promoter sequence (from $-2,735$ to +104 ) from mouse genomic DNA by PCR using the following primers: 5'-ACTCTCGAGCCAGGCACTCTTTCTCGACT-3' and 5'-AGTAAGCTTGGAGTCTCCTGCACGCTAGT-3'. We generated a series of Trb3 promoterLUC constructs carrying deletions within the Trb3 promoter region (Trb3-2.4 kLuc, -1.7 kLuc, -1.1 kLuc, and -340 bLuc). To generate Trb3 -360 Dmut construct, we used the QuikChange II Site-Directed Mutagenesis Kit (Stratagene). We used the following PCR primers to generate mutations of the consensus forkhead binding motifs in the Trb3 -340 promoter region: F1, 5'GGGATTGACGAGATTTTTACAGAGCCTCCGTTCATTCTTTTTTC-3'; R1, 5'-GAAAAAAGAATGAACGGAGGCTCTGTAAAAATCTCGTCAATCCC-3'; F2, 5'-CTATTCTCATTTCATATACGGGGACAGAGGTATAAAGTACTACAATACCG-3'; and R2, 5'-CCGTATTGTAGTACTTTATACCTCTGTCCCCGTATATGAAATGAGAATAG-3'. To generate DBD-FoxO1ADA, we substituted Asn208 and His212 in the DNA binding domain for Ala and Arg, respectively, using the QuikChange II Site-Directed Mutagenesis Kit (Stratagene). We confirmed the mutations by DNA sequencing.

Transient transfection and luciferase assay. We seeded SV40-transformed hepatocytes in 12-well plates at a density of $10^{5}$ cells/well. One day after plating, cells were transfected by Lipofectamine 2000 (Invitrogen) according to the manufacturer's protocol. We carried out cotransfections with Trb3 promoter-reporter constructs (50 ng) and control pCMV5 vector and/or pCMV5-WT FoxO1 or pCMV5-FoxO1ADA. The total amount of DNA was adjusted to $1.25 \mu \mathrm{g} /$ well with control vector DNA. For adenoviral infection, 5 hours after transfection we added non-protein-coding adenovirus, adenovirus encoding FoxO1ADA or WT FoxO1. Ten hours after transfection, we changed the medium to Ham F-12 (Invitrogen) supplemented with $0.5 \%$ BSA, FSK $(10 \mu \mathrm{M})$, DEX $(100 \mathrm{nM})$, insulin $(100 \mathrm{nM})$, or LY294002 $(30 \mu \mathrm{M})$. After 30 hours, we washed cells with ice-cold PBS and harvested them. We carried out luciferase assays with Dual Luciferase Reporter Assay System (Promega) and quantified luciferase activity using Monolight 3010 Luminometer (BD Bioscience). As an internal standard to normalize for transfection efficiency, we cotransfected pRL-CMV Renilla luciferase control vector (5 ng) (Promega). All experiments were performed in triplicate and at least twice. For siRNA experiments, we cotransfected either FoxO1siRNA (sc-35383; Santa Cruz Biotechnology Inc.) or control siRNA (sc-37007; Santa Cruz Biotechnology Inc.) with Trb3 promoter reporter construct at a final concentration of $30 \mathrm{nM}$. Forty-eight hours after transfection, we performed luciferase assays.

Animal experiments. We used 7-week-old C57BL/6 male mice (Charles River Laboratories). We delivered recombinant adenovirus encoding FoxO1ADA or nonrecombinant protein coding adenovirus by tail vein injection at a dose of $4 \times 10^{9}$ virus particles/g (vp/g) body weight in a total volume of $0.25 \mathrm{ml}$. The total viral load was approximately $0.8 \times 10^{11} \mathrm{vp} /$ mouse. We carried out the experiments 5 days after injection. We fixed livers in $10 \%$ formaldehyde and analyzed by oil red O staining to evaluate hepatic lipid content. We measured hepatic TG contents as described previously (7). We used different enzymatic assays for plasma chemistry analyses, including plasma glucose (Invitrogen), plasma insulin (Mercodia), plasma TGs (Wako), total cholesterol (Wako), and $\beta$-hydroxybutyrate (Ranbut; Randox). All experiments were approved by the Columbia University Animal Care and Use Committee.

ChIP assays. We performed ChIP assays in hepatocytes as described previously (48). We used the following primers for the Trb3 promoter: $5^{\prime}$-TCGAACTCAACGATCCACCT-3' and 5'-GGAGTCTCCTGCACGCTAGT- 3 '.

Statistics. Values are expressed as mean \pm SEM. Significant differences between mean values were evaluated using 2-tailed, unpaired Student's $t$ test (when 2 groups were analyzed) or 1-way ANOVA followed by StudentNewman-Keuls test (for 3 groups).

\section{Acknowledgments}

We thank M. Montminy for the anti-Trb3 antiserum and members of the Accili laboratory for critical comments. This work was supported by NIH grants DK57539, DK58282, and DK63608 (to the Columbia University Diabetes and Endocrinology Research Center) and grants from the American Diabetes Association, the Uehara Memorial Foundation Fellowship, and the Astellas Foundation Fellowship for Research on Metabolic Disorders (to M. Matsumoto).

Received for publication October 4, 2005, and accepted in revised form June 16, 2006.

Address correspondence to: Domenico Accili, Department of Medicine, Columbia University Medical Center, 1150 St. Nicholas Avenue, New York, New York 10032, USA. Phone: (212) 851-5332; Fax: (212) 851-5331; E-mail: da230@columbia.edu.
1. Accili, D. 2004. Lilly lecture 2003: the struggle for mastery in insulin action: from triumvirate to republic. Diabetes. 53:1633-1642.

2. Cherrington, A.D. 1999. Banting lecture 1997. Control of glucose uptake and release by the liver in vivo. Diabetes. 48:1198-1214.

3. Schwarz, J.M., Linfoot, P., Dare, D., and Aghajanian, K. 2003. Hepatic de novo lipogenesis in normoinsulinemic and hyperinsulinemic subjects consuming high-fat, low-carbohydrate and low-fat, high-carbohydrate isoenergetic diets. Am. J. Clin. Nutr. 77:43-50.

4. Iozzo, P., et al. 2004. Defective liver disposal of free fatty acids in patients with impaired glucose tolerance. J. Clin. Endocrinol. Metab. 89:3496-3502.

5. Ginsberg, H.N., Zhang, Y.L., and Hernandez-Ono, A. 2005. Regulation of plasma triglycerides in insulin resistance and diabetes. Arch. Med. Res. 36:232-240.

6. Shimomura, I., et al. 2000. Decreased IRS-2 and increased SREBP-1c lead to mixed insulin resis- tance and sensitivity in livers of lipodystrophic and ob/ob mice. Mol. Cell. 6:77-86.

7. Matsumoto, M., et al. 2002. Role of the insulin receptor substrate 1 and phosphatidylinositol 3-kinase signaling pathway in insulin-induced expression of sterol regulatory element binding protein $1 \mathrm{c}$ and glucokinase genes in rat hepatocytes. Diabetes. 51:1672-1680.

8. Wolfrum, C., Asilmaz, E., Luca, E., Friedman,J.M., and Stoffel, M. 2004. Foxa2 regulates lipid metabolism 
and ketogenesis in the liver during fasting and in diabetes. Nature. 432:1027-1032.

9. Zhang, L., Rubins, N.E., Ahima, R.S., Greenbaum, L.E., and Kaestner, K.H. 2005. Foxa2 integrates the transcriptional response of the hepatocyte to fasting. Cell Metab. 2:141-148.

10. Accili, D., and Arden, K.C. 2004. FoxOs at the crossroads of cellular metabolism, differentiation, and transformation. Cell. 117:421-426.

11. Nakae, J., et al. 2002. Regulation of insulin action and pancreatic beta-cell function by mutated alleles of the gene encoding forkhead transcription factor Foxo1. Nat. Genet. 32:245-253.

12. Puigserver, P., et al. 2003. Insulin-regulated hepatic gluconeogenesis through FOXO1-PGC-1alpha interaction. Nature. 423:550-555.

13. Altomonte, J., et al. 2004. Foxo1 mediates insulin action on apoC-III and triglyceride metabolism. J. Clin. Invest. 114:1493-1503. doi:10.1172/ JCI200419992.

14. Herzig, S., et al. 2003. CREB controls hepatic lipid metabolism through nuclear hormone receptor PPAR-gamma. Nature. 426:190-193.

15. Ogg, S., et al. 1997. The Fork head transcription factor DAF-16 transduces insulin-like metabolic and longevity signals in C. elegans. Nature. 389:994-999.

16. Lin, K., Dorman, J.B., Rodan, A., and Kenyon, C. 1997. daf-16: an HNF-3/forkhead family member that can function to double the life-span of Caenorhabditis elegans. Science. 278:1319-1322.

17. Ide, T., et al. 2004. SREBPs suppress IRS-2-mediated insulin signalling in the liver. Nat. Cell Biol. 6:351-357.

18. Zhang, J., et al. 2001. Insulin inhibits transcription of IRS-2 gene in rat liver through an insulin response element (IRE) that resembles IREs of other insulin-repressed genes. Proc. Natl. Acad. Sci. U. S. A. 98:3756-3761.

19. Dong, X., et al. 2006. Irs 1 and Irs2 signaling is essential for hepatic glucose homeostasis and systemic growth. J. Clin. Invest. 116:101-114. doi:10.1172/JCI25735.

20. Nakae, J., Kitamura, T., Silver, D.L., and Accili, D. 2001. The forkhead transcription factor Foxo1 (Fkhr) confers insulin sensitivity onto glucose-6phosphatase expression. J. Clin. Invest. 108:1359-1367. doi:10.1172/JCI200112876.

21. Miyake, K., et al. 2002. Hyperinsulinemia, glucose intolerance, and dyslipidemia induced by acute inhibition of phosphoinositide 3-kinase signaling in the liver. J. Clin. Invest. 110:1483-1491. doi:10.1172/JCI200215880
22. Zhang, W., et al. 2006. FOXO1 regulates multiple metabolic pathways in the liver: effects on gluoconeogenic, glycolytic and lipogenic gene expression. J. Biol. Chem. 281:10105-10117.

23. Altomonte, J., et al. 2003. Inhibition of Foxo1 function is associated with improved fasting glycemia in diabetic mice. Am. J. Physiol. Endocrinol. Metab. 285:E718-E728.

24. Aoyama, T., et al. 1998. Altered constitutive expression of fatty acid-metabolizing enzymes in mice lacking the peroxisome proliferator-activated receptor alpha (PPARalpha). J. Biol. Chem. 273:5678-5684.

25. Leone, T.C., Weinheimer, C.J., and Kelly, D.P. 1999. A critical role for the peroxisome proliferator-activated receptor alpha (PPARalpha) in the cellular fasting response: the PPARalpha-null mouse as a model of fatty acid oxidation disorders. Proc. Natl. Acad. Sci. U. S. A. 96:7473-7478.

26. Semenkovich, C.F. 1997. Regulation of fatty acid synthase (FAS). Prog. Lipid Res. 36:43-53.

27. Tang, E.D., Nunez, G., Barr, F.G., and Guan, K.L. 1999. Negative regulation of the forkhead transcription factor FKHR by Akt. J. Biol. Chem. 274:16741-16746.

28. Liu, Z.P., Wang, Z., Yanagisawa, H., and Olson, E.N. 2005. Phenotypic modulation of smooth muscle cells through interaction of Foxo 4 and myocardin. Dev. Cell. 9:261-270.

29. Rother, K.I., et al. 1998. Evidence that IRS-2 phosphorylation is required for insulin action in hepatocytes. J. Biol. Chem. 273:17491-17497.

30. Kerouz, N.J., Horsch, D., Pons, S., and Kahn, C.R 1997. Differential regulation of insulin receptor substrates-1 and - 2 (IRS-1 and IRS-2) and phosphatidylinositol 3-kinase isoforms in liver and muscle of the obese diabetic (ob/ob) mouse. J. Clin. Invest. 100:3164-3172

31. Lizcano, J.M., and Alessi, D.R. 2002. The insulin signalling pathway. Curr. Biol. 12:R236-R238.

32. Du, K., Herzig, S., Kulkarni, R.N., and Montminy, M. 2003. TRB3: a tribbles homolog that inhibits Akt/PKB activation by insulin in liver. Science. 300:1574-1577.

33. Koo, S.H., et al. 2004. PGC-1 promotes insulin resistance in liver through PPAR-alpha-dependent induction of TRB-3. Nat. Med. 10:530-534.

34. Schwarzer, R., Dames, S., Tondera, D., Klippel, A., and Kaufmann, J. 2006. TRB3 is a PI 3-kinase dependent indicator for nutrient starvation. Cell. Signal. 18:899-909.

35. Puig, O., Marr, M.T., Ruhf, M.L., and Tjian, R. 2003. Control of cell number by Drosophila FOXO: downstream and feedback regulation of the insulin receptor pathway. Genes Dev. 17:2006-2020

36. Hashimoto, T., et al. 2000. Defect in peroxisome proliferator-activated receptor alpha-inducible fatty acid oxidation determines the severity of hepatic steatosis in response to fasting. J. Biol. Chem. 275:28918-28928.

37. Brunet, A., et al. 2004. Stress-dependent regulation of FOXO transcription factors by the SIRT1 deacetylase. Science. 303:2011-2015.

38. Kitamura, Y.I., et al. 2005. FoxO1 protects against pancreatic beta cell failure through NeuroD and MafA induction. Cell Metab. 2:153-163.

39. Nakae, J., Kitamura, T., Ogawa, W., Kasuga, M., and Accili, D. 2001. Insulin regulation of gene expression through the forkhead transcription factor Foxo1 (Fkhr) requires kinases distinct from Akt. Biochemistry. 40:11768-11776.

40. Nakae, J., Barr, V., and Accili, D. 2000. Differential regulation of gene expression by insulin and IGF-1 receptors correlates with phosphorylation of a single amino acid residue in the forkhead transcription factor FKHR. EMBO J. 19:989-996.

41. Vicent, D., et al. 2003. The role of endothelial insulin signaling in the regulation of vascular tone and insulin resistance. J. Clin. Invest. 111:1373-1380. doi:10.1172/JCI200315211.

42. Sam, S., and Dunaif, A. 2003. Polycystic ovary syndrome: syndrome XX? Trends Endocrinol. Metab. 14:365-370.

43. Taylor, S.I. 1992. Lilly Lecture: molecular mechanisms of insulin resistance. Lessons from patients with mutations in the insulin-receptor gene. Diabetes. 41:1473-1490.

44. Withers, D.J., et al. 1998. Disruption of IRS-2 causes type 2 diabetes in mice. Nature. 391:900-904

45. Previs, S.F., Withers, D.J., Ren, J.M., White, M.F., and Shulman, G.I. 2000. Contrasting effects of IRS-1 versus IRS- 2 gene disruption on carbohydrate and lipid metabolism in vivo. J. Biol. Chem. 275:38990-38994.

46. Hribal, M.L., Nakae, J., Kitamura, T., Shutter, J.R., and Accili, D. 2003. Regulation of insulin-like growth factor-dependent myoblast differentiation by Foxo forkhead transcription factors. J. Cell Biol. 162:535-541.

47. Kitamura, T., et al. 1999. Insulin-induced phosphorylation and activation of cyclic nucleotide phosphodiesterase 3B by the serine-threonine kinase Akt. Mol. Cell. Biol. 19:6286-6296.

48. Nakae, J., et al. 2003. The forkhead transcription factor foxo1 regulates adipocyte differentiation. Dev. Cell. 4:119-129. 